\title{
Use of a live vaccine to modulate Cryptobia salmositica infections in Salmo salar
}

\author{
Christina M. Davy ${ }^{1,2}$, Erika Crispo ${ }^{1,3}$, Patrick T. K. Woo ${ }^{1, *}$ \\ ${ }^{1}$ Department of Integrative Biology, University of Guelph, Guelph, Ontario N1G 2W1, Canada \\ ${ }^{2}$ Present address: Department of Biology, University of Western Ontario, 1151 Richmond St. N., London, \\ Ontario N6A 5B7, Canada \\ ${ }^{3}$ Present address: McGill University, Redpath Museum, 859 Sherbrooke St. W., Montréal, Québec H3A 2K6, Canada
}

\begin{abstract}
The vaccine strain of Cryptobia salmositica multiplies in Atlantic salmon Salmo salar. and it can modulate the severity of the disease in Cryptobia-infected individuals. Fish injected with the vaccine $3 \mathrm{~d}$ post-infection with $C$. salmositica had lower peak parasitaemias and higher antibody titres than infected fish given the vaccine $7 \mathrm{~d}$ post-infection or those infected fish that were not given the vaccine.
\end{abstract}

KEY WORDS: Cryptobia salmositica $\cdot$ Vaccine $\cdot$ Immune response $\cdot$ Atlantic salmon $\cdot$ Salmo salar Resale or republication not permitted without written consent of the publisher

\section{INTRODUCTION}

Cryptobiosis is caused by the haemoflagellate Cryptobia salmositica Katz, 1951. Clinical signs of cryptobiosis include exophthalmia, splenomegaly, abdominal distension, ascites fluid, anaemia (Woo 1979), and anorexia (Thomas \& Woo 1992, Chin et al. 2004). The pathogen has been found in all species of Pacific Oncorhynchus occurring along the west coast of North America, and it is transmitted in streams and rivers by the freshwater leech Piscicola salmositica (Woo 2003).

The parasite secretes a $200 \mathrm{kDa}$ metalloprotease, and this is an important disease-causing factor in salmonid cryptobiosis (Zuo \& Woo 1997). An attenuated strain of Cryptobia salmositica has been developed (Woo \& Li 1990), and it does not produce/secrete the metalloprotease (Zuo \& Woo 1997). The strain has remained avirulent for the last $16 \mathrm{yr}$ and is used routinely as an experimental vaccine because it does not cause disease and circulates in the blood for at least 6 mo (e.g. Woo \& Li 1990, Li \& Woo 1995). Also, it does not multiply readily in trout, but confers protection for up to $2 \mathrm{yr}$ (Li \& Woo 1995). However, the attenuated strain multiplies in Atlantic salmon Salmo salar (Ardelli \& Woo 2002). We suggest that the vaccine, when injected into infected Atlantic salmon, will provide additional immunological stimulation to modulate infection by the pathogenic strain, and this is the purpose of the current study.

\section{MATERIALS AND METHODS}

Forty adult Atlantic salmon (average weight $255.9 \pm$ 74.8 g.; average length, $284.1 \pm 31.2 \mathrm{~cm}$ ) from the Ministry of Natural Resources, Ontario, Canada, were used. They were held in four $125 \mathrm{l}$ circular fiberglass tanks (10 fish tank ${ }^{-1}$ ) at $10^{\circ} \mathrm{C}$, with a photoperiod of $16 \mathrm{~h}$ light:8 h dark. Fish were fed to satiation twice daily with sinking trout pellets (Martin Feed Mills), and water was re-circulated to maintain similar water conditions in all tanks.

The pathogenic Cryptobia salmositica used in this study was originally obtained from the leech Piscicola salmositica. It has been cloned and is maintained by serial passage in rainbow trout (Woo 1979). The vaccine strain (Woo \& Li 1990) was maintained at $10^{\circ} \mathrm{C}$ in Minimum Essential Medium, supplemented with L-glutamine, Hanks' salts, 25 mM HEPES buffer, and $25 \%$ heat-inactivated fetal bovine serum. 
There were 4 groups (A to D) and each had 10 fish. The lengths $(p=0.308)$ and weights $(p=0.826)$ of fish were not significantly different between groups (1-way ANOVA). Fish in one control group (Group A) were injected intraperitoneally (i.p.) with the vaccine (100000 parasites in $0.1 \mathrm{ml}$ PBS [phosphate buffered saline; $\mathrm{pH} 7$.2] fish ${ }^{-1}$ ) from cultures, while those in the second control group (Group B) were injected i.p. with the pathogen (50000 parasites in $0.1 \mathrm{ml} \mathrm{PBS}$ fish $^{-1}$ ) from an infected trout. The 2 treatments groups (Groups C and D) were also infected. with the pathogen $\left(50000\right.$ fish $\left.^{-1}\right)$. Fish in Group C were injected i.p with the vaccine (100000 fish $\left.{ }^{-1}\right) 3 \mathrm{~d}$ post-infection (d.p.i), while fish in Groups A, B and D were each injected with $0.1 \mathrm{ml}$ PBS. At 7 d.p.i., fish in Group D were injected with the vaccine (100000 per fish ${ }^{-1}$ ) while those in Groups A, B and C were injected with PBS. This was done to ensure that all the fish were exposed to similar handling and inoculation stresses.

Blood was taken at $1 \mathrm{wk}$ post-infection (w.p.i) and then weekly for $8 \mathrm{wk}$. Fish were anaesthetized in 2-phenoxyethanol, and blood $\left(0.5 \mathrm{ml} \mathrm{fish}{ }^{-1}\right)$ was drawn from the caudal vein using a needle (25 gauge) and a syringe that had been rinsed with heparinized saline. Blood $(20 \mu \mathrm{l})$ from each fish was blotted onto Whatman \#5 filter paper and air-dried. The filter papers were stored at $-20^{\circ} \mathrm{C}$ until needed for antibody determination by enzyme-linked immunosorbent assay (ELISA).

Parasites were counted using a haemocytometer after the blood was with diluted with PBS (Archer 1965). If no parasites were detected, the blood was examined for Cryptobia using the haematocrit centrifuge technique (Woo \& Wehnert 1983). The number of pathogens per fish from the 2 treatment groups (Groups C and D) for a particular week was derived by subtracting the average number of attenuated parasites (Group A) from the number of parasites in the $\mathrm{C}$ or $\mathrm{D}$ group fish. The packed cell volume (PCV) was the average of duplicates for each fish at each bleed (Woo 1979).

Antibody response (as optical density or OD value) was determined using ELISA (Sitja-Bobadilla \& Woo 1994). The antigen was from cultures of the pathogen, and the second antibody (rabbit anti-salmon immunoglobulin was diluted 1:1000 in dilution buffer) was provided by K. Buchmann, Royal Veterinary and Agricultural University, Denmark. Three replicates were made with serum elute (eluted in PBS from dried blood on filter paper) for each fish, and controls included wells without the third antibody (peroxidase-conjugated goat antirabbit immunoglobulin diluted 1:1000 in dilution buffer; Sigma Chemical), and wells with blood blots from uninfected fish that were inoculated with PBS. An ELISA plate (Sarstedt) was used for samples from each week, and 3 replicates without the third-antibody and naïve controls were included in each plate.
The OD values in the wells were read at $492 \mathrm{~nm}$ using a MAXline Microplate Reader (Molecular Devices), with the software SOFTmax v. 2.01. The average OD value for the no-third-antibody control for each plate was subtracted from each of the other OD values in the same plate. The average OD value from each fish was used in the analysis.

STATISTICA v. 7.1 (StatSoft) was used for statistical analyses, and all tests were at 0.05 level of significance. Unpaired $t$-tests were used to compare parasitaemias and PCV between infected-only (Group B) and vaccine-only (Group A) controls, and to compare OD values between vaccine-only (Group A) and naïve controls. For these $t$-tests, we tested for the equality of variances (Levene's test) and used the appropriate $\mathrm{p}$-values from the $t$-tests depending on whether or not variances were equal. Kruskal-Wallis tests were used to examine the difference between treatments (Groups C and D) and infected-only controls (Group B) with respect to parasite numbers, packed cell volume, and corrected OD at each of $6 \mathrm{wk}$. Post-hoc multiple comparisons (2-tailed) were used to determine which pairs of treatments differed significantly for each week.

\section{RESULTS}

Fish mortalities occurred and they were caused either by cryptobiosis, or by furunculosis (which occurred during the study), or a combined effect of the 2 diseases. At 1 w.p.i., one fish died in each of the 2 treatments (Groups C and D). Between 4 to 5 w.p.i., one more fish died in Group C, 2 more in Group D, 5 more in Group B, and 2 in Group A. Between 5 to 6 w.p.i., 1 more died in each of Groups C and D, 1 died in Group B, and 2 died in Group A.

Cryptobia salmositica was detected in all groups at 1 w.pi. using the haematocrit centrifuge technique; however, it was not detected using the haemocytometer until 2 w.p.i. in Groups B, C and D, and not until 3 w.p.i. in Group A. Group B had higher parasitaemias than Group A only at 1 w.p.i. $(p=0.0041), 2$ w.p.i. $(p=$ $0.0048)$, and 4 w.p.i. ( $p=0.0011)$. The parasitaemias (Fig. 1a) in all 4 groups peaked at 4 w.p.i. At 1 to 4 w.p.i., Group C had significantly lower parasitaemias than Group B, whereas Group D was not significantly different from Group B (Table 1, Fig. 1a). There were no significant differences between the treatment groups (Groups C and D) the control group (Group B) in the other weeks (Table 1, Fig. 1a).

PCV was significantly higher in Group A than in Group B at 3 w.p.i. ( $p=0.002)$, and close to significantly higher at 4 w.p.i. $(p=0.052)$, while this was not the case in other weeks $(0.134<\mathrm{p}<0.710)$. Group $\mathrm{C}$ 

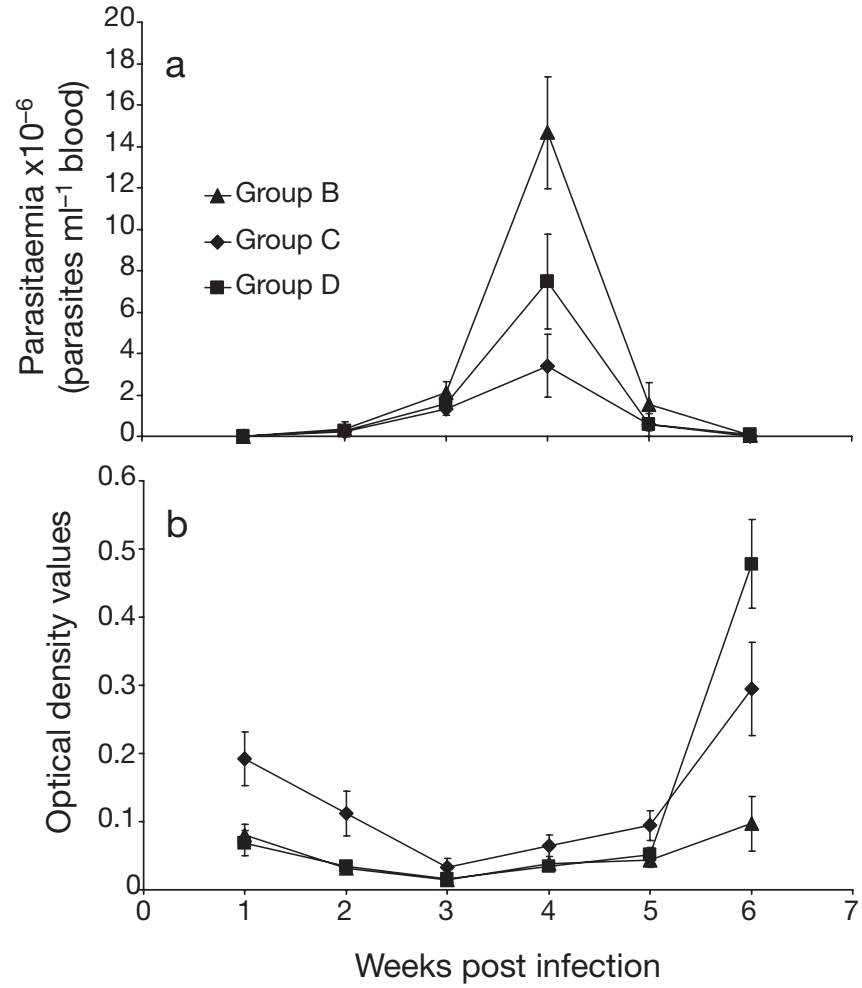

Fig. 1. Cryptobia salmositica. (a) Parasites present in fish blood over 6 wk for 3 treatments (B, C and D); (b) antibody responses (optical density, ELISA test) to same treatments. Values are means \pm SE. Treatment B: each fish was infected with 50000 pathogenic $C$. salmositica, and injected with PBS (phosphate buffered saline) at 3 and at $7 \mathrm{~d}$ post-infection (d.p.i.). Treatment C: each fish was infected with 50000 pathogenic C. salmositica, injected with the vaccine (100000 attenuated C. salmositica per fish) at 3 d.p.i., and injected with PBS at 7 d.p.i. Treatment D: each fish was infected with 50000 pathogenic C. salmositica, injected with PBS at 3 d.p.i., and with the vaccine $\left(100000\right.$ fish $\left.^{-1}\right)$ at 7 d.p.i.

had higher PCV than Group D only at 2 w.p.i. (Table 1). Since furunculosis also causes anaemia, there is no way to determine the severity of the anaemia caused by the Cryptobia salmositica.

At 2 w.p.i., the corrected OD values (OD minus the average $\mathrm{OD}$ of the no-third-antibody controls) for

Table 1. Cryptobia salmositica. p-values obtained from Kruskal-Wallis tests comparing treatment and controls for parasitaemias, packed-cell volume (PCV), and optical density. p.i.: post-infection $;$-: no data. ${ }^{*}$ Significant at $\mathrm{p}<0.05$

\begin{tabular}{|cccc|}
\hline Week p.i. & Parasitaemia & PCV & Optical density \\
\hline 1 & $0.0218^{*}$ & 0.3370 & 0.2551 \\
2 & 0.3906 & $0.0042^{*}$ & $0.0028^{*}$ \\
3 & 0.7831 & 0.1490 & 0.3671 \\
4 & $0.0246^{*}$ & 0.0983 & 0.1501 \\
5 & 0.7668 & 0.7369 & 0.0619 \\
6 & 0.2748 & - & $0.0129^{*}$ \\
\hline
\end{tabular}

Group C were higher than those for Group B and Group D (Table 1, Fig. 1b). Group D had higher OD values than Group B at 6 w.p.i. (Table 1, Fig. 1b). There were no significant differences between treatments and control in other weeks (Table 1, Fig. 1b), or between corrected OD values in vaccinated controls and naïve fish $(0.235<\mathrm{p}<0.65)$.

\section{DISCUSSION}

Our current study confirms that the vaccine strain multiplies in Atlantic salmon, and that post-infection vaccination of Cryptobia salmositica-infected Atlantic salmon with the live vaccine may have a modulating effect if it is administered soon after infection. Salmon vaccinated $3 \mathrm{~d}$ after infection had lower peak parasitaemias and higher antibody titres than infected fish that were given the vaccine $7 \mathrm{~d}$ post-infection, or those that were not given the vaccine. We conclude the additional stimulation of the immune system early in the disease process (e.g. $3 \mathrm{~d}$ after infection) by the vaccine (multiplying parasites stimulates higher production of antibodies) is sufficient, and this helps with lowering the peak parasitaemia and in the recovery. Atlantic salmon are also quite susceptible to furunculosis, and outbreaks occur in hatcheries. In addition, both humoral and cell-mediated responses are important in protection (Hiney \& Oliver 1999). Since all 4 groups of fish were infected with Aeromonas salmonicida, we suggest the differences (in parasitaemias and antibody production against C. salmositica) between Group C and the other 2 groups (B and D) would have been similar in the absence of Aeromonas infection. Furthermore, without furunculosis, there would have been no mortality in the vaccine only group (Group A). More extensive studies are required to explore and refine this approach as an option for the control of cryptobiosis. This study also confirms earlier reports (e.g. Li \& Woo 1995, Aredelli \& Woo 2002, Chin et al. 2004) that the humoral response to the vaccine is an important component in protection against cryptobiosis.

Acknowledgements. We thank M. Eldridge, J. Pootoolal, and A. Chin for technical assistance, and B. Robinson and T. Nudds for help with the statistical design. The research was funded by a Natural Science and Engineering Council (Canada) grant to P.T.K.W.

\section{LITERATURE CITED}

Archer RK (1965) Haematological techniques for use on animals. Blackwell Scientific Publications, Oxford

Ardelli BF, Woo PTK (2002) Experimental Cryptobia salmositica (Kinetoplastida) infections in Atlantic salmon (Salmo salar): cell-mediated and humoral immune response 
against the pathogenic and vaccine strains of the parasite. J Fish Dis 25:265-274

Chin A, Guo FC, Bernier NJ, Woo PTK (2004) Effect of Cryptobia salmositica-induced anorexia on feeding behavior and immune response in juvenile rainbow trout Oncorhynchus mykiss. Dis Aquat Org 58:17-26

Hiney M, Oliver G (1999) Furunculosis (Aeromonas salmonicida). In: Wood PTK (ed) Fish diseases and disorders, Vol 3. Viral, bacterial and fungal infections. CABI Publishing, Wallingford, p 341-425

Li S, Woo PTK (1995) Efficacy of a live Cryptobia salmositica vaccine, and the mechanism of protection in vaccinated rainbow trout, Oncorhynchus mykiss, against cryptobiosis. Vet Immunol Immunopathol 48:343-353

Sitja-Bobadilla AS, Woo PTK (1994) An enzyme-linked immunosorbent assay (ELISA) for the detection of antibodies against the pathogenic haemoflagellate, Cryptobia salmositica Katz, and protection against cryptobiosis in juvenile rainbow trout, Oncorhyncus mykiss (Walbaum), inoculated with a live vaccine. J Fish Dis 17:399-408

Editorial responsibility: Dieter Steinhagen,

Hannover, Germany
Thomas PT, Woo PTK (1992) Anorexia in Oncorhynchus mykiss infected with Cryptobia salmositica (Sarcomastigophora: Kinetoplastida): its onset and contribution to the immunodepression. J Fish Dis 15:443-447

Woo PTK (1979) Trypanoplasma salmositica: experimental infections in rainbow trout, Salmo gairdneri. Exp Parasitol 47:36-48

Woo PTK (2003) Cryptobia (Trypanoplasma) salmositica and salmonid cryptobiosis. J Fish Dis 26:627-646

Woo PTK, Li S (1990) In vitro attenuation of Cryptobia salmositica and its use as a live vaccine against cryptobiosis in Oncorhynchus mykiss. J Parasitol 76:752-755

Woo PTK, Wehnert S (1983) Direct transmission of a haemoflagellate, Cryptobia salmositica Katz, 1951 (Kinetoplastida: Bodonina) between rainbow trout under laboratory conditions. J Protozool 39:334-337

Zuo X, Woo PTK (1997) Purified metallo-protease from the pathogenic haemoflagellate Cryptobia salmositica and its in vitro proteolytic activities. Dis Aquat Org 30: 177-185

Submitted: February 2, 2006; Accepted: December 21, 2006 Proofs received from author(s): May 14, 2007 